\title{
Beam Dynamics through the CONCERT-ESS Linac
}

\author{
N. Pichoff*, R. Duperrier, D. Uriot, CEA/DSM/DAPNIA/SEA, France
}

\begin{abstract}
The CONCERT concept aims to deliver high power pulsed beams to a various number of applications : mater studies with spallation neutrons, waste transmutation, exotic beam production, irradiation tools... It is one of the extension of the European Spallation Source project (ESS) dedicated to spallation neutrons production for mater studies. Both protons and $\mathrm{H}$ - have to be accelerated and guided to each target. Two $50 \mathrm{~mA} \mathrm{H}^{-}$branches are funnelled with one $100 \mathrm{~mA} \mathrm{H} \mathrm{H}^{+}$branch at around 23.5 $\mathrm{MeV}$. A low energy $\mathrm{H}^{-}$branch is constituted of one RFQ from around $50 \mathrm{keV}$ to $2 \mathrm{MeV}$, a chopper line (for ring injection at high energy), an other RFQ to $5 \mathrm{MeV}$, and a DTL to the funnel line. The low energy $\mathrm{H}+$ is constituted of one RFQ from around $95 \mathrm{keV}$ to $5 \mathrm{MeV}$ and a DTL to the funnel line. The beams are then accelerated through a SDTL, a CCL and a Superconducting cavity Linac (SCL). The goal of this paper is to present the results of beam dynamics calculations through the CONCERT linac.
\end{abstract}

\section{INTRODUCTION}

The specificity of the CONCERT concept is that its accelerator has to be able to accelerate both $\mathrm{H}^{-}$and $\mathrm{H}^{+}$ beams. The $\mathrm{H}^{-}$beam is injected into a ring (stripped to protons) and compressed to a $650 \mathrm{~ns}$ high intensity pulse. It is used to create short spallation neutrons pulses for mater study (ESS project). The $\mathrm{H}^{+}$beam is used for the others applications: long spallation neutrons pulse, waste transmutation, exotic beam production, irradiation tools... For consideration of power, $100 \mathrm{~mA}$ beams are needed. The $\mathrm{H}^{-}$pulsed is about $1 \mathrm{~ms}$ long, 70\% chopped (@2.05MeV), with $50 \mathrm{~Hz}$ repetition rate. As 5\% dutycycle $\mathrm{H}^{-}$sources, with emittance lower $0.3 \pi$.mm.rad and high reliability are highly challenging at $100 \mathrm{~mA}$ peak current, it has been chosen to funnel two $50 \mathrm{~mA} \mathrm{H}$ beams lines at about $23.5 \mathrm{MeV}$ [1]. Because high duty-cycle $(>5 \%)$ are challenging even at $50 \mathrm{~mA}$ for $\mathrm{H}^{-}$sources, $\mathrm{H}^{+}$ line is necessary for the other applications.

After the funnel line, $\mathrm{H}^{-}$and $\mathrm{H}^{+}$beams are accelerated to $1.34 \mathrm{GeV}$ in the same structures : Separated Drift Tube Linac (SDTL); Coupled-Cavity Linac (CCL); Superconductive Cavity Linac (SCL).

\section{LINAC BASELINE}

The low energy part [2] (until the SDTL) is represented on figure 1. The RF frequency of each structure as well as the transition energies are given.

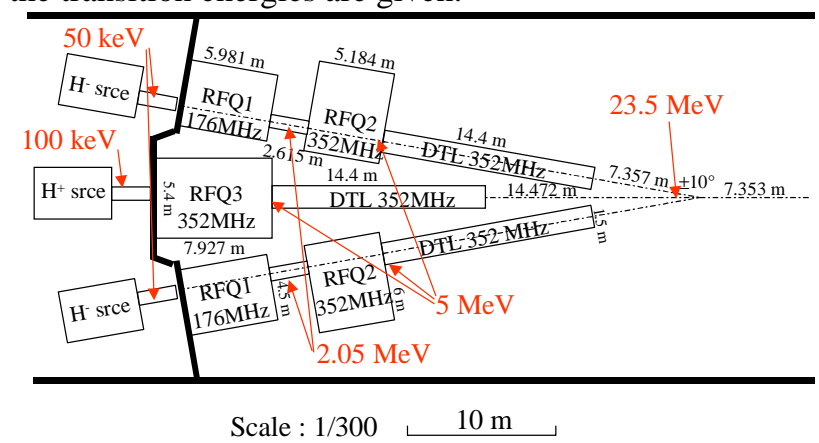

Figure 1: Low energy baseline.

The $23.5 \mathrm{MeV}$ beams are then injected in a $352.2 \mathrm{MHz}$ separated DTL (SDTL). This kind of structure allows an easier alignment of quadrupoles than in classical DTL as they stand outside the small DTL tanks. Moreover it allows an easier matching. The doublet lattice scheme is used. The beam is then accelerated to $90 \mathrm{MeV}$. At this energy 24-cells $704 \mathrm{MHz}$ coupled-cavities replace the 5cells drift-tube cavities, as their shunt impedance becomes higher. The doublet focusing scheme is kept for an easier matching. The beam is then accelerated to 186 $\mathrm{MeV}$. At this energy, 5-cells 704.4 MHz superconducting cavities are used. The first section contains 14 cryomodules with $3 \beta=0.658$ cavities. It goes to 430 $\mathrm{MeV}$. The second section contains 24 cryomodules with 4 $\beta=0.846$ cavities. It goes to $1.334 \mathrm{GeV}$. In each cavity the peak magnetic field has been limited to $50 \mathrm{mT}$ which corresponds to about $23.5 \mathrm{MV} / \mathrm{m}$ peak electric field. the superconducting sections are described in a companion paper [3].

\section{MATCHING}

In beam dynamics driven by space-charge, it is now well known that mismatching is one of the main halo source observed in simulations [4]. A good matching between the structures is then very important. It is achieved in 3 stages :

- Try to keep the same focusing scheme (here, doublet) in each section,

- Keep the phase advance per meter continuous through the linac,

\footnotetext{
"Email: nicolas.pichoff@cea.fr
} 
- Match the Courant-Snyder parameters of the channel through the transitions by adjusting quadrupoles and cavities strength (beam rms matching).

The $\mathrm{H} / \mathrm{H}^{+}$common channel has been matched using TraceWIN [5] code with $\mathrm{H}^{-}$beam. This matching has been kept for $\mathrm{H}^{+}$beam.

Because of different source performances, the initial $\mathrm{H}$ beam normalised transverse rms-emittance is assumed to be $0.3 \pi . \mathrm{mm}$.mrad as it is only $0.25 \pi . \mathrm{mm}$.mrad for $\mathrm{H}^{+}$ beam. The longitudinal emittances at the RFQ exits are different because the bunching RFQ frequencies are different $\left(176.1 \mathrm{MHz}\right.$ for $\mathrm{H}^{-}$and $352.2 \mathrm{MHz}$ for $\mathrm{H}^{+}$).

Multiparticle simulations have been done, in the RFQ with TOUTATIS code [6], and with PARTRAN code and the PICNIC 3D space-charge routine [7] in the rest of the linac.

On figure 2 are represented the $\mathrm{H}^{+}$beam envelopes in $\mathrm{X}$ and longitudinal directions from the exit of the last RFQ. The matching, even if it has been done with $\mathrm{H}^{-}$beam, is very correct.

CEA Saclay - DSM/DAPNIA/SEA
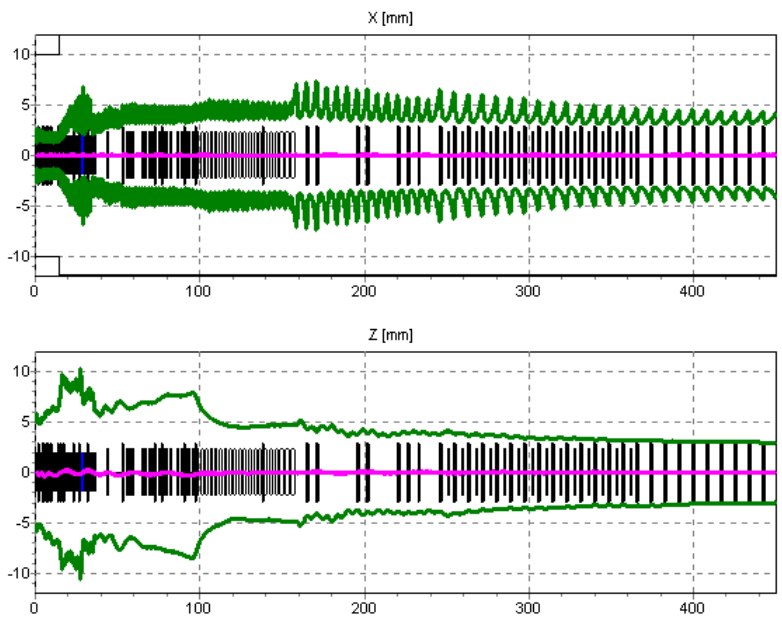

Figure 2: $\mathrm{H}^{+}$transverse and longitudinal envelopes.

The rms emittance growth and the halo parameter [8] evolutions of both beams are presented on figures 3 and 4. The main emittance growth and halo formation happen in the funnel line.

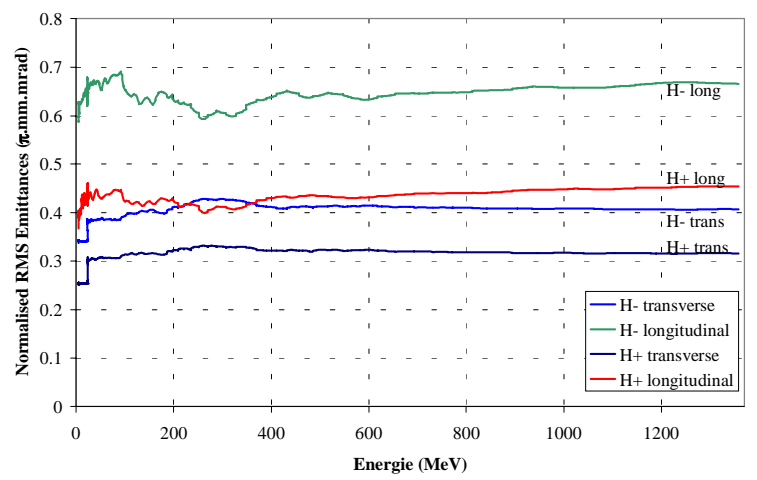

Figure 3: $\mathrm{H}^{+}$and $\mathrm{H}^{-}$rms emittances evolution.

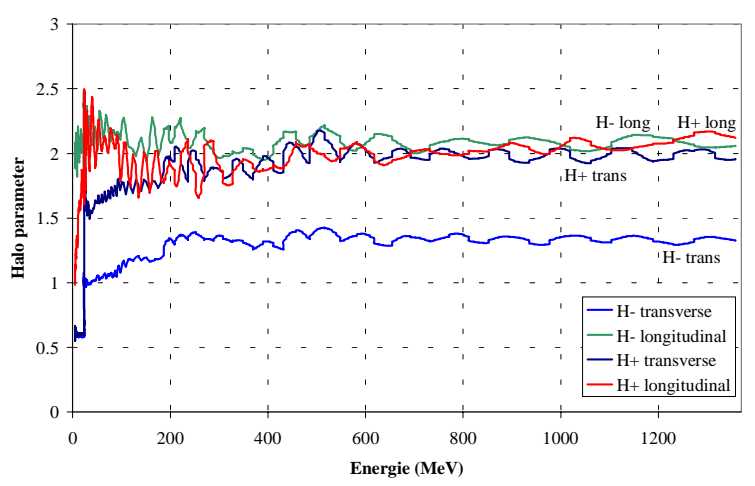

Figure 4: $\mathrm{H}^{+}$and $\mathrm{H}^{-}$halo parameters.

The output phase-space distributions $\left(\mathrm{H}^{-}\right.$on figure 5 and $\mathrm{H}^{+}$on figure 6) exhibits a small but negligible halo.
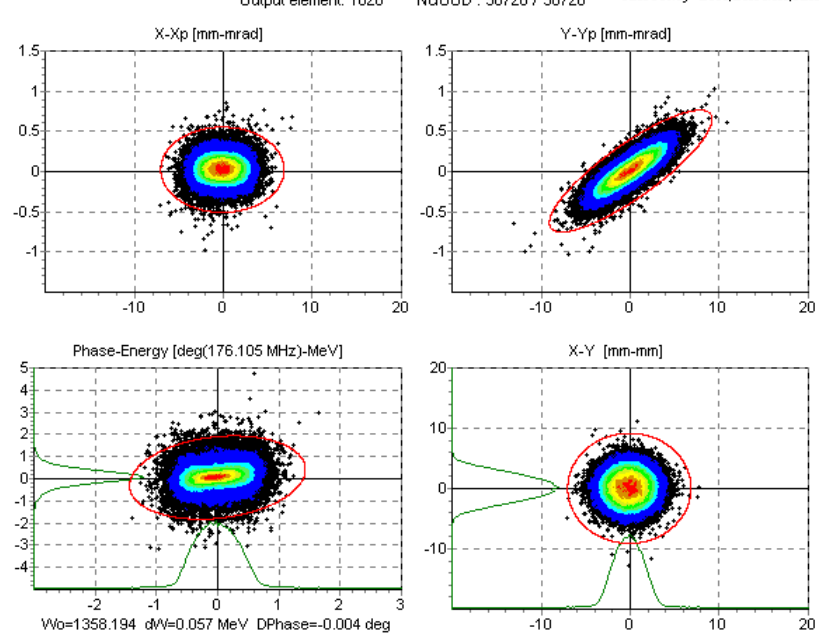

Figure 5: $\mathrm{H}$ output phase-space distribution. In red are the 20 times the rms-emittance ellipses.
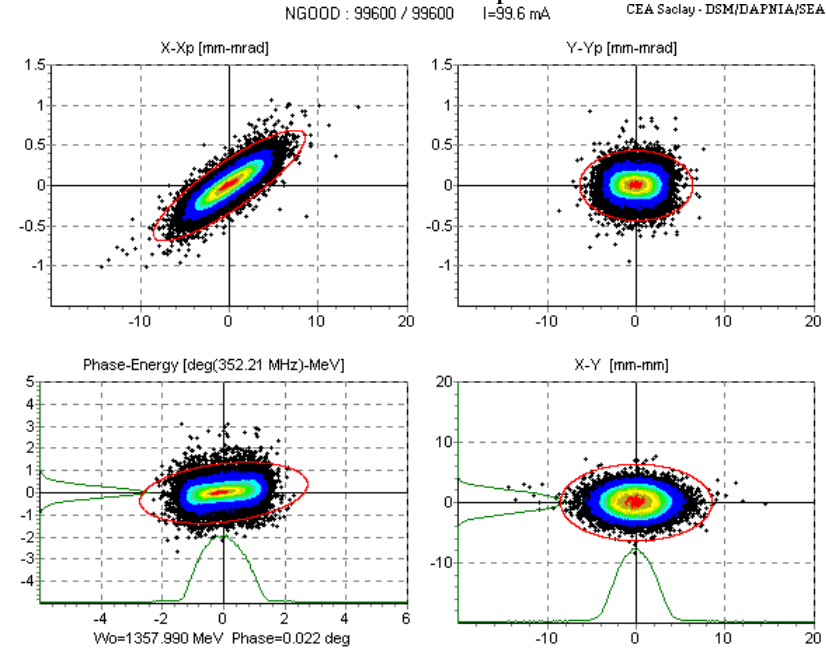

Figure 6: $\mathrm{H}^{+}$output phase-space distribution. In red are the 20 times the rms-emittance ellipses. 


\section{MISMATCHED BEAM}

To test the linac "robustness", a simulation with a $20 \%$ initially mismatched has been done. This mismatch has been introduced in RFQ exit by increasing the $\beta$ and $\alpha$ beam Twiss parameters by a $1.2^{2}$ factor in all directions. The evolution of rms emittances is plotted on figures 6 . An initial small emittance growth occurs. As the beam matches itself to the channel through space-charge forces, their is no more emittance growth in the high energy part.

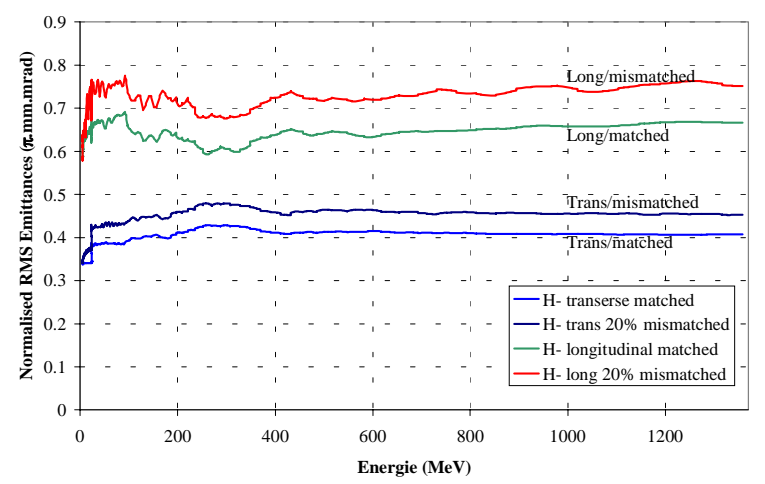

Figure 6 : Emittances of matched and 20\% initially mismatched $\mathrm{H}^{-}$beam.

\section{QPOLE GRADIENTS ERRORS}

In order to set the tolerances on quadrupole gradients, a statistical study with a variable quadrupole gradient error amplitude $\mathrm{A}_{\mathrm{g}}$ has been done. A set of 500 different linacs with $\mathrm{A}_{\mathrm{g}}$ varying from 0 to $1 \%$ (each quad has a gradient error between $-\mathrm{A}$ and $+\mathrm{A}$, with a uniform probability distribution) has been simulated with $\mathrm{H}^{+}$. The induced extra emittance growth has been plotted as a function of $\mathrm{A}_{\mathrm{g}}$ on figure 7. No matching correction scheme has been implemented as we don't know at that time how it could be measured.

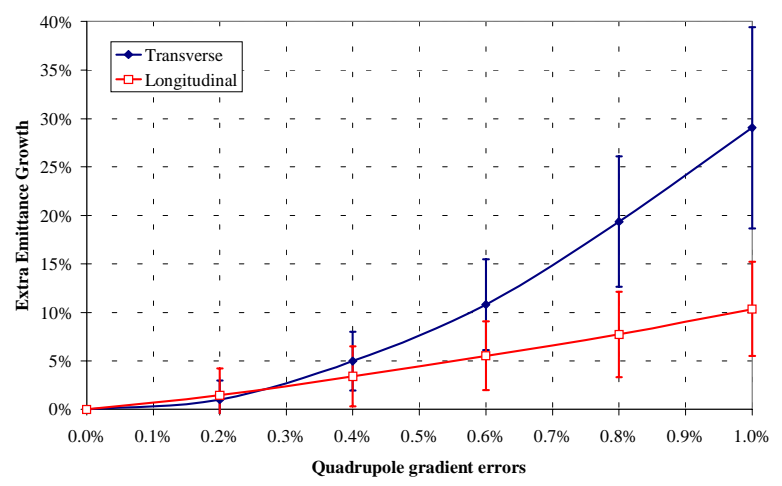

figure 7 : Extra emittance growth as a function of quadrupole gradient amplitude error.

A tolerance on quadrupole gradients lower than $0.2 \%$ will be certainly sufficient.

\section{CONCLUSION}

Both $\mathrm{H}^{-}$and $\mathrm{H}^{+}$CONCERT-ESS linacs have been designed. The matching of each line has been calculated. The matching of common part have been calculated with $\mathrm{H}^{-}$and applied successfully with $\mathrm{H}^{+}$beam. End-to-end simulations, with a water-bag matched distribution at RFQs entrance, have been done. The "robustness" test consisting of injecting a $20 \%$ mismatched beam at the linac input has been passed. An error studies on quadrupole gradients have been done giving a $0.2 \%$ tolerance (or less). An other study have exhibited that a $1 \%$ error on superconducting cavities field and a $1^{\circ}$ on their phases gives $700 \mathrm{keV}$ and a $1^{\circ}$ (@704.4Mhz) standard deviations on the final energy and beam phase, with no losses. Other errors studies should be investigated, from which an efficient measurement/correction scheme should to be found.

\section{REFERENCES}

[1] N. Pichoff et al., "The ESS-CONCERT funnel line, this proc.

[2] R. Ferdinand et al., "Low energy part of the CONCERT high-power proton linac", this proc.

[3] A. Mosnier et al.,"SC Proton Linac Design for CONCERT Multi-Users Facility", this proc.

[4] T.P. Wangler et al., "Dynamics of Beam Halo in Mismatched Beams", Proc. of Linac96, Geneva, August 26-30, 1996.

[5] D. Uriot, N. Pichoff, "TraceWIN Documentation" DSM/DAPNIA/SEA local note 2000/45.

[6] R. Duperrier, "Toutatis, a radio-frequency quadrupole code", Phys. Rev, Spec. Top. Acc. \& Beams, December 2000.

[7] N. Pichoff et al., Simulation Results with an Alternate $3 D$ Space Charge Routine, PICNIC, Proc. of Linac98, Chicago, August 23-28, 1998.

[8] C.K. Allen, T.P. Wangler, "A parameter for quantifying beam halo formation in phase space", LA-UR-00-4952, October 2000. 